\title{
Encoches transverses sur la pars basilaris de l'os occipital : nouveaux cas chez de très jeunes immatures
}

\section{Transverse clefts on the pars basilaris of the occipital bone : new cases in very young immature}

\author{
M. NIEL ${ }^{1}$, C. RIGEADE ${ }^{1,2}$, K. CHAUMOITRE ${ }^{1,3}$, S. TZORTZIS ${ }^{1,4}$, M. SIGNOLI ${ }^{1}$, Y. ARDAGNA ${ }^{1}$, P. \\ ADALIAN $^{1}$ \\ ${ }^{1}$ Aix Marseille Univ, CNRS, EFS, ADES, Marseille, France \\ 2 Inrap Méditerranée, Centre de recherches archéologiques de Marseille, 14 rue d'Anthoine - 13002 Marseille, \\ France \\ ${ }^{3}$ Service de Radiologie et Imagerie médicale, Hôpital Nord, CHU Marseille, Assistance Publique des Hôpitaux de \\ Marseille, Chemin des Bourrely, Marseille cedex 20 13915, France \\ ${ }^{4}$ Direction Régionale des Affaires Culturelles Provence-Alpes-Côte d'Azur, 23 boulevard du Roi René, 13617 Aix- \\ en-Provence Cedex 1, France
}

NIEL Mélissa

0491698890

melissaniel@hotmail.fr 


\section{Résumé}

But de l'étude : Des encoches transverses peuvent apparaître sur la pars basilaris de l'os occipital. L'objectif est de calculer la prévalence de ces encoches, de manière à caractériser l'impact potentiel de cette anomalie sur l'estimation de l'âge au décès, puis de questionner la diversité de forme et la longueur des encoches en fonction du statut sain ou pathologique du très jeune immature étudié.

Matériel et méthodes: Deux échantillons sont utilisés; le premier est un échantillon d'imagerie médicale incluant 482 CT scans de fœtus et de nourrissons (pathologiques et non pathologiques) âgés de 13 semaines in utero à 15 mois. Le second est un échantillon ostéoarchéologique composé de 134 fœtus et immatures provenant de sites archéologiques français. Les fréquences pour chacun des échantillons sont calculées et comparées aux données de la littérature, puis la longueur des encoches est mesurée et interprétée.

Résultats : La fréquence des encoches observées dans nos échantillons est faible. II apparait en imagerie médicale que les encoches sont 2,9 fois plus fréquentes chez les individus pathologiques que chez les individus sains, sans toutefois pouvoir conclure à une différence statistiquement significative, et peuvent être aussi bien unilatérales que bilatérales avec un ratio entre la longueur des encoches et la largeur de la pars basilaris qui semble plus important.

Conclusion : Cette étude apporte de nouvelles connaissances sur les encoches transverses. Bien qu'elle ne permette pas de trancher sur leur étiologie, la découverte de ce type d'anomalie doit faire réfléchir sur la présence d'une éventuelle pathologie risquant de biaiser l'estimation de l'âge au décès.

Mots-clés: Morphologie osseuse ; variation osseuse asymptomatique ; syndrome pathologique ; origine génétique ; embryologie ; étiologie 


\begin{abstract}
Aim of the study: Transverse notches can appear on the pars basilaris of the occipital bone. The objective is to calculate the prevalence of these notches to characterize the potential impact of this anomaly on age-at-death estimation and to question the diversity of appearance and length of the notches according to the normal or pathological status of very young immatures.

Material and methods: Two samples are used: a medical imaging sample including 482 CT scans of fetuses and infants (pathological and healthy) aged 13 weeks in utero to 15 months; and a bioarchaeological sample of 134 fetuses and immatures from French archaeological sites. The frequencies of pars basilaris notches are calculated for each sample and compared with literature data, and the notches length is measured and interpreted.

Results: The frequencies of the notches observed in our samples are low. In the medical imaging, notches are 2.9 times more frequent for pathological individuals than healthy individuals; but this difference is not statistically significant, and can be unilateral as well as bilateral with higher ratio between notch length and the width of the pars basilaris.

Conclusion: This study brings new insight on transverse notches of the pars basilaris. Although it does not allow to conclude on their etiology, the discovery of this type of anomaly should question the possibility of pathologies likely to skew age at death estimates.
\end{abstract}

Keywords : Bone morphology; asymptomatic bone variation; pathological syndrome; genetic origin; embryology; etiology 


\section{Introduction}

Dans les cas de découvertes d'ossements de très jeunes immatures, au-delà de la nécessité d'identifier chacune des portions ossifiées des os en cours de formation, il est souvent difficile de déceler la présence d'une éventuelle anomalie de développement. Toutefois leur détection peut s'avérer fondamentale en contexte judiciaire, puisqu'elle permet d'évaluer le taux de confiance que nous pourrons donner aux estimations de l'âge au décès qui seront établies. En effet, de nombreuses anomalies de développement ont une répercussion sur la croissance biométrique, et les méthodes d'estimation d'âge se basant sur des mesures osseuses pourraient se voir invalidées.

Dans ce travail, nous proposons une mise au point concernant l'os occipital, et plus particulièrement la pars basilaris de l'os en cours de formation. Puis nous nous focalisons sur un signe potentiel d'anomalie de développement : l'existence d'encoches transverses. Nous cherchons à identifier leur diversité de forme (unilatérale ou bilatérale; longueur et longueur relative) et questionnons leur étiologie à partir d'un échantillon d'imagerie médicale incluant aussi bien des individus pathologiques que non pathologiques. Enfin, nous évaluons leur prévalence sur un échantillon ostéo-archéologique dont les restes osseux sont issus de cinq sites archéologiques du sud de la France.

Chez le fœtus, l'occipital est composé de quatre éléments distincts; une pars squama, deux pars lateralis et une pars basilaris (également nommée basioccipital ou processus basilaire). Ces quatre éléments forment les limites du foramen magnum; la pars squama postérieurement, les pars lateralis latéralement, et la pars basilaris antérieurement (Figure 1).

\section{FIGURE 1}

Figure 1 Vue externe de l'os occipital d'un fœtus âgé de 26 semaines de grossesse.

Le centre d'ossification 
enchondrale de la pars basilaris apparaît entre la $11^{\text {ème }}$ et la $12^{\text {ème }}$ semaine de vie prénatale (1-8) à partir du cartilage parachordal. L'ossification débute de la partie antérieure du cartilage vers la partie postérieure (4), avec une expansion rapide au début, qui ralentit après 15 semaines (8). La pars basilaris s'articule antérieurement avec le sphénoïde au niveau de la synchondrose sphéno-occipitale, avec une fusion entre 11 et 16 ans chez les filles et 13 et 18 ans chez les garçons $(7,9)$. Postérieurement, elle s'articule avec les pars lateralis au niveau de la suture intra-occipitalis antérieure (Figure 1), avec une fusion entre 5 et 7 ans $(7,10,11)$. La pars basilaris devient reconnaissable à partir du $3^{\text {ème }}$ mois. Os fin initialement allongé avec une base antérieure arrondie, sa forme se complexifie progressivement avec l'âge $(1,7)$. D'aspect triangulaire, sa forme devient quadrangulaire en fin de grossesse avec une base antérieure carrée et des angles latéraux qui se développent à partir du $7^{\text {ème }}$ mois (7). A terme, la face exocrânienne ou inférieure est assez plate tandis que la face endocrânienne est légèrement concave avec des foramens nourriciers en surface. La surface antérieure est relativement plate, tandis que la surface postérieure est courbée avec deux cornes sur lesquelles se trouve un tiers de la structure anatomique qui formera les condyles occipitaux (Figure 2). Enfin, les angles latéraux sont composés d'une partie antérieure qui s'articule avec la pars petrosa de l'os temporal, et d'une partie postérieure qui s'articule avec les processus jugulaires et condylaires des 
pars lateralis (7). A l'union du tiers antérieur et des deux tiers postérieurs de la face inférieure de la pars basilaris se trouvent le tubercule pharyngien sur lequel s'insère le ligament longitudinal antérieur (12). Ce tubercule n'est pas souvent visible chez les jeunes individus, mais l'est vers l'âge de 2 ou 3 ans (7). Juste en avant de ce tubercule se trouve la fossette pharyngienne qui est une partie légèrement déprimée, qui marque le passage de la notochorde $(7,12)$ (Figure 2 ).

\section{FIGURE 2}

Figure 2 La pars basilaris illustrée en vue inférieure d'un individu périnatal (extrait et modifié de Scheuer \& Black, 2004, p.74).

Des anomalies de développement entraînant une modification de sa forme peuvent apparaître sur la pars basilaris de l'os occipital. On peut observer sur celle-ci une division complète dans le sens longitudinal, transversal ou simultanément dans les deux directions. La division peut aussi être incomplète sous la forme d'une encoche latérale ou bilatérale de dimensions et de forme variables, au niveau du tubercule pharyngien (13-17). Ces encoches sont assez rares, le plus souvent asymétriques (14) et ont été mentionnées comme étant la fissure de Sauser (Sauser's fissure) décrite pour la première fois radiologiquement dans l'atlas de List (16). Dans la littérature ancienne et notamment dans son traité des variations des os du crâne (14), Le Double dresse un inventaire de cas rencontrés au XIXème siècle. L'auteur fait par exemple mention de segmentation transversale complète chez des fœtus hémicéphales cyclopes et non cyclopes, chez un " aliéné » italien, chez un adolescent de 16 ans acrocéphale, ou encore chez un fœtus hydrocéphale. Environ 10 cas sur 4312 crânes sont répertoriés 
par Le Double, ce qui en fait un caractère rarissime $(0,23 \%)$. Les encoches incomplètes unilatérales ou bilatérales sont plus fréquentes. Elles ont été répertoriées au XIXème siècle pour 25 cas sur 496 crânes ( 21 fois des deux côtés, 2 fois à droite, 2 fois à gauche), soit 5,04\%. Dans son atlas, Barnes illustre lui aussi plusieurs types d'encoches (13). Cette anomalie morphologique, aussi bien présente sur des sujets immatures que sur des sujets adultes, est observée au sein de plusieurs populations ostéo-archéologiques issues de diverses périodes chronologiques $(14,15,17-22)$.

La partie osseuse détachée du reste de la pars basilaris, ou antérieure à la fissure, est appelée le basiotique $(17,22)$, le basicoccipital antérieur ou encore le prébasioccipital (21), tandis que la partie postérieure est nommée le basioccipital $(14,17,20,23,24)$. Les encoches ont de nombreuses dénominations; elles sont appelées incisures basi-prébasioccipitales $(23,24)$, fissures transverses $(25,26)$, encoches transverses $(20,27,28)$, encoches basioccipitales (15), encoches basilaires (19), fissures transverses basilaires (17), ou encore fissure basilaire transverse (11). Nous retenons pour cette étude les dénominations basiotique, basioccipital et encoches transverses. Chez le fœtus et jusqu'à l'adolescence, le basiotique est rattaché au basioccipital par une couche de cartilage $(13-15,20)$, qui s'ossifie chez l'adulte pour former une fissure ou une suture entre ces deux parties (14). L'origine de ces encoches transverses n'est pas certaine mais se produit probablement à un stade très précoce du développement embryologique (27), et trois hypothèses sont formulées dans la littérature : elles seraient une simple variation anatomique asymptomatique, elles seraient le signe 
d'un développement pathologique, ou alors elles seraient d'origine génétique.

L'objectif de notre travail est dans un premier temps de calculer la prévalence de ces encoches, de manière à caractériser l'impact potentiel de ce signe sur les estimations de l'âge au décès. Puis dans un second temps, en questionnant la diversité de présentation et la longueur des encoches en fonction de pathologies identifiées sur les individus de la collection d'imagerie médicale, notre objectif est de voir s'il est possible de privilégier l'une des causes potentielles évoquées dans la littérature.

\section{Matériel et méthodes}

\section{Echantillons d'étude}

Deux types d'échantillons incluant de très jeunes immatures ont été utilisés pour cette étude. Le premier est un échantillon d'imagerie médicale dont les individus ont passé un examen CT scan ante mortem ou post mortem dans les Hôpitaux de Marseille (France). II comprend 482 CT scans de fœetus et de nourrissons (316 individus non pathologiques et 166 individus pathologiques) âgés de 13 semaines in utero à 15 mois post partum. Le classement des individus pathologiques/non pathologiques a été établi par des examens multidisciplinaires ante mortem (CT scan in utero) et des examens post mortem conduits par des experts des centres pluridisciplinaires de diagnostic prénatal. En effet, depuis la création de ces centres (Décret 97-578 du 28 Mai 1997 consolidée le 11 Mai 2018), les fœtus sont systématiquement examinés en cas d'interruption médicale de grossesse ou de mort spontanée (fausse couche et mort in utero). Ces examens permettent d'identifier les malformations osseuses ou viscérales, les anomalies chromosomiques, et 
parfois de préciser les causes de la mort. Pour les individus postnataux, les CT scan sont prescrits en cas de mort inattendue ou subite du nourrisson, d'accident de la voie publique, ou pour appuyer les enquêtes médico-légales. Dans cet échantillon d'étude, les individus ont été considérés comme "non pathologiques» en absence de malformation externe ou viscérale, absence d'anomalie osseuse et cérébrale, présence d'un caryotype normal, absence de diabète ou d'hypertension artérielle maternel, absence d'antécédent de maladie congénitale maternelle).

Le second est un échantillon ostéo-archéologique composé de 134 fœtus et très jeunes immatures dont l'âge estimé par les méthodes biométriques osseuses (longueurs diaphysaires) et dentaire (éruption dentaire) (29-33) s'étend de la période fœtale à 4 ans. Les individus proviennent de cinq sites archéologiques français : Chapelle Notre-Dame de la mer à Fos-sur-Mer (Provence-Alpes-Côte d'Azur, ensemble funéraire du VIİ̀me au XVIII ème siècle) (34), 110 Chemin de la Farigoule à Castelnau-le-Lez (Occitanie, échantillon issu du cimetière médiéval du XIIème au XVIème siècle et d'une occupation d'habitat protohistorique) (35), Chemin de Saint-Privat à Rousset (Provence-Alpes-Côte d'Azur, daté du Xème au XIIème siècle) (36), Eglise Saint-Pierre à Moirans (Auvergne-Rhône-Alpes, daté de la période antique à la période moderne) (37), et Chapelle Saint-Jean à L'Argentière-la-Bessée (Provence-AlpesCôte d'Azur, daté de la période médiévale à la période moderne) (38).

\section{Acquisition des données}

Les examens d'imagerie médicale ont été collectés sur le PACS (Picture Archiving and Communication System, McKesson Medical Imaging Group, Richmond, BC, Canada) à l'Hôpital Nord de Marseille. Les individus ont été examinés avec 
un CT scan hélicoïdal (Somatom Sensation Cardiac 64; Siemens, Erlangen, Germany) dont les paramètres sont les suivants : voltage 100-140 kVp, ampérage 50-180 mAs, 512x512 pixels, resolution $0,25-4,87$ pixels $/ \mathrm{mm}$, taille de voxel de $0,5 \times 0,5 \times 0,6$ ou $1 \mathrm{~mm}^{3}$, épaisseur de coupe de 0,6 à $1 \mathrm{~mm}$. Les coupes de haute résolution enregistrées en format DICOM (Digital Imaging and Communications in Medicine) ont été anonymisées avant d'être utilisées dans cette étude.

Avant de reconstruire le plus fidèlement possible les surfaces osseuses des pars basilaris en trois-dimensions (3D), une valeur de niveau de gris ou valeur seuil représentant les unités Hounsfield, et qui correspond à la séparation entre les os et les tissus mous ou I'air, a été calculée à partir des coupes DICOM avec le logiciel ImageJ ${ }^{\circledR} 1.43$ (National Institutes of Health, USA). La valeur seuil a été obtenue en calculant la valeur seuil moyenne (ou Threshold Mean Value) (39), qui est une moyenne des valeurs de demi-hauteur maximale (ou Half Maximum Height) (40). La TMV obtenue pour chaque os a été renseignée sur le logiciel Avizo Standard Edition (v.7.0.0 ${ }^{\circledR}$, Visualization Sciences Group, SAS) permettant ainsi de reconstruire les surfaces osseuses en $3 D$ fidèles à la réalité.

Les pars basilaris des individus de l'échantillon ostéo-archéologique ont quant à elles été observées à l’œil nu.

Etude des encoches transverses : comment et pourquoi ?

Dans un premier temps, et afin de juger de leur caractère rare ou fréquent, nous avons observé les pars basilaris une à une, puis calculé la fréquence des encoches transverses 
unilatérales et bilatérales pour chacun des échantillons (imagerie médicale et ostéoarchéologique). Ces fréquences ont ensuite été comparées aux données issues de la littérature. La comparaison entre les échantillons a été testée statistiquement en calculant la probabilité exacte de Fisher à l'aide du logiciel de statistique $R^{\circledR}$ (version 3.4.2) (41).

Dans un second temps, la longueur des encoches a été mesurée via l'outil de mesure du logiciel Avizo $^{\circledR}$ pour les surfaces reconstruites en $3 \mathrm{D}$, ou avec un pied à coulisse digital sur les os secs, pour voir s'il existait une différence entre les individus sains et pathologiques. Toutes les mesures sont exprimées en millimètres.

\section{Résultats}

\section{Fréquence des encoches transverses}

Chez les 482 individus de l'échantillon d'imagerie médicale, on retrouve au total cinq encoches transverses, dont quatre unilatérales (droite ou gauche) et une bilatérale, soit une fréquence de 1,04\% (Tableau 1, Figure 3). Plus en détails, il en existe une fréquence de $0,63 \%$ pour les individus non pathologiques et de 1,81\% pour les individus pathologiques. Tous les cas d'encoches retrouvés ici concernent uniquement des fœtus ( 3 filles et 2 garçons).

Pour les 134 individus de l'échantillon ostéo-archéologique, on observe un total de deux encoches transverses bilatérales soit une fréquence de 1,49\% (Tableau 1, Figure 3).

Bien que la fréquence d'apparition des encoches retrouvée sur les individus pathologiques soit supérieure à celle retrouvée sur les individus sains dans l'échantillon d'imagerie médicale, il n'existe pas de différence significative au seuil de $5 \%(p=0,3453)$. De la même manière, il n'existe pas de différence significative entre 
cette fréquence et celle retrouvée sur les individus de l'échantillon ostéo-archéologique $(p=1)$. Enfin, le test de Fisher ne met pas non plus en évidence de différence significative entre la fréquence des individus porteurs d'encoches de l'échantillon d'imagerie médicale total (sains et pathologiques) et celle des individus porteurs d'encoches de l'échantillon ostéo-archéologique $(p=0,6495)$.

\section{Morphologie des pars basilaris}

Les individus de l'échantillon d'imagerie médicale ayant des pars basilaris avec encoches unilatérales ont environ tous le même âge ; entre 22 et 25 semaines de grossesse (Tableau 2). Les morphologies osseuses devraient donc être proches. C'est le cas, sauf pour la pars basilaris de l'individu IM-327 qui semble avoir un développement morphologique moins avancé que les autres : le bord antérieur du foramen magnum n'est pas très profond et la base antérieure de la pars basilaris est plutôt arrondie. L'individu IM-397 présente une encoche bilatérale asymétrique, tandis que celles observées chez les individus archéologiques sont plutôt symétriques, et ces deux individus ont une morphologie de pars basilaris semblable.

Toutes les encoches transverses sont situées au même endroit sur la pars basilaris, c'est-àdire juste en dessous des angles latéraux, au niveau du tubercule pharyngien (Figure 2 et 3 ).

\section{Comparaison des fréquences avec la littérature}

Les encoches transverses de la pars basilaris ont été étudiées sur différentes populations et périodes chronologiques qui permettent d'en retrouver les fréquences. Quelques études ont été résumées dans le Tableau $2(18-20,23-25,42)$. Les fréquences de nos deux échantillons (imagerie médicale: 1,04\%; ostéo-archéologique : 1,49\%) sont cohérentes 
Tableau 1 Fréquence des encoches unilatérales et bilatérales dans l'échantillon d'imagerie médicale (IM) et l'échantillon

ostéo-archéologique (OA).

n Encoche unilatérale Encoche bilatérale Fréquence (\%)

$\begin{array}{llll}\text { Individus sains (IM) } & 316 & 2 & 0,63\end{array}$

Individus pathologiques (IM) 166

2

81

Individus archéologiques (OA) 134

$1 \quad 1,81$




\section{FIGURE 3}

Figure 3 Illustration en vue exocrânienne des encoches unilatérales et bilatérales sur les sept pars basilaris de nos deux échantillons.

avec les données de la littérature (Tableau 2) : leur fréquence est plutôt faible. A partir du Tableau 2, nous avons recalculé la fréquence de l'ensemble des échantillons d'immatures (issues de la littérature et de cette étude) et d'adultes (issues de la seule littérature), puis nous les avons comparés. II existe une différence significative au seuil de $5 \%$ entre les immatures et les adultes, toutes périodes chronologiques confondues $(p=0,0011)$ : les encoches sont plus fréquentes chez les immatures que chez les adultes. Enfin, il n'existe pas de différence significative entre les fréquences des immatures issues de la littérature et les immatures de cette étude $(p=0,2165)$.

\section{Mesures des encoches}

Dans l'échantillon d'imagerie médicale, les fœtus ont des encoches dont la longueur varie entre 0,94 à $3,92 \mathrm{~mm}$ (Tableau 3). Les encoches des pars basilaris des individus de l'échantillon ostéo-archéologique âgés de [0-1] an mesurent entre 6,03 et 7,12 $\mathrm{mm}$. Le ratio entre la longueur des encoches et la largeur des pars basilaris a été calculé. En cas d'encoches bilatérales, la somme de l'encoche droite et gauche a été prise en compte (Tableau 3). Il apparait que les ratios vont de 0,11 à 0,58 et que les encoches peuvent mesurer plus de la moitié de la largeur totale des pars basilaris, ce qui se retrouve dans les deux cas d'encoches bilatérales de l'échantillon ostéo-archéologique.

\section{Discussion}

\section{Origine embryologique des encoches}

Ces encoches transverses ont amené les anatomistes à rediscuter le nombre de centres d'ossification de la pars basilaris. Il est admis qu'elle est 
Tableau 2 Tableau récapitulatif de quelques études concernant des cas d'encoches tranverses de la pars basilaris chez l'immature et l'adulte pour différentes populations et périodes chronologiques.

\begin{tabular}{|c|c|c|c|c|c|c|}
\hline Source & Population & Période & $\begin{array}{c}\text { Nombre de } \\
\text { cas }\end{array}$ & Fréquence & Immature & Adulte \\
\hline $\begin{array}{c}\text { Kawakubo et al. } \\
\text { (2014) }\end{array}$ & Japonaise & $5500-2800 \mathrm{cal} \mathrm{BP}$ & $2 / 12$ & $16,67 \%$ & $x$ & \\
\hline Smith (1912) & Egyptienne & Egypte antique & $1 / 62$ & $1,61 \%$ & $x$ & \\
\hline Anderson (2000) & Italienne & Médiévale & $2 / 75$ & $2,66 \%$ & $x$ & \\
\hline Limson (1932) & $\begin{array}{l}\text { Euro-Afro- } \\
\text { Américaine }\end{array}$ & $1930^{\prime}$ & $3 / 163$ & $1,84 \%$ & $x$ & \\
\hline Kruyff (1967) & Canadienne & $1960^{\prime}$ & $1 / 80$ & $1,25 \%$ & $x$ & \\
\hline $\begin{array}{c}\text { Kawakubo et al. } \\
(2014)\end{array}$ & Japonnaise & $5500-3300 \mathrm{cal} \mathrm{BP}$ & $3 / 71$ & $4,23 \%$ & & $x$ \\
\hline Smith (1912) & Egyptienne & Egypte antique & $0 / 1038$ & $0 \%$ & & $x$ \\
\hline Osawa (1894) & Japonnaise & $1890^{\prime}$ & $1 / 162$ & $0,62 \%$ & & $x$ \\
\hline Shima (1941) & Mongole & $1940^{\prime}$ & $2 / 193$ & $1,04 \%$ & & $x$ \\
\hline $\begin{array}{l}\text { Kawakubo et al. } \\
\text { (2014) }\end{array}$ & Japonnaise & $2010^{\prime}$ & $1 / 156$ & $0,64 \%$ & & $x$ \\
\hline
\end{tabular}


Tableau 3 Age (SG=semaines de grossesse), sexe (F=féminin, $M=$ =masculin, I=indéterminé), développement, type et longueur des encoches en fonction des individus, et ratio entre la longueur des encoches et la largeur des pars basilaris.

\begin{tabular}{|c|c|c|c|c|c|c|c|}
\hline Individu & Age & Sexe & Développement & $\begin{array}{l}\text { Encoche } \\
\text { G (mm) }\end{array}$ & $\begin{array}{l}\text { Encoche } \\
D(\mathrm{~mm})\end{array}$ & $\begin{array}{l}\text { Largeur } \\
\text { pars } \\
\text { basilaris } \\
(\mathrm{mm})\end{array}$ & $\begin{array}{c}\text { Encoche(s) } \\
\text { /largeur }\end{array}$ \\
\hline \multicolumn{8}{|c|}{ Echantillon d'imagerie médicale } \\
\hline IM-327 & 22 SG & $\mathrm{F}$ & $\begin{array}{c}\text { Pathologique : } \\
\text { anomalie crâne et } \\
\text { membres }\end{array}$ & & 0,94 & 8,42 & 0,11 \\
\hline IM-170 & $23 \mathrm{SG}$ & M & $\begin{array}{c}\text { Pathologique : } \\
\text { association de type } \\
\text { VACTER }\end{array}$ & 3,92 & & 10,04 & 0,39 \\
\hline IM-159 & $23 \mathrm{SG}$ & $\mathrm{F}$ & Sain & 1,63 & & 8,01 & 0,20 \\
\hline IM-166 & 24 SG & $\mathrm{F}$ & Sain & 1,74 & & 9,28 & 0,19 \\
\hline IM-397 & 25 SG & M & $\begin{array}{l}\text { Pathologique: } \\
\text { anomalie cérébrale }\end{array}$ & 2,94 & 1,00 & 10,51 & 0,38 \\
\hline \multicolumn{8}{|c|}{ Echantillon ostéo-archéologique } \\
\hline CAS-2109 & $0-1$ an & 1 & Indéterminé & 6,36 & 7,12 & 23,39 & 0,58 \\
\hline FSM-290 & $0-1$ an & I & Indéterminé & 6,03 & 6,38 & 22,58 & 0,55 \\
\hline
\end{tabular}


formée par la fusion du cartilage parachordal formant la plaque basale, et de quatre sclérotomes occipitaux qui forment la partie postérieure $(11,12,28,43-46)$. L'unique centre d'ossification enchondrale de la pars basilaris apparaît entre la $11^{\text {ème }}$ et la $12^{\text {ème }}$ semaine de vie prénatale (1-8). Certains auteurs pensent que les encoches transverses seraient la résultante non pas d'un unique centre d'ossification mais de deux, qui n'auraient pas complètement fusionnés. Le premier serait à l'origine du basiotique, et le second à l'origine du basioccipital. La limite entre ces deux éléments osseux correspond à la crista synostotica (17), qui serait la ligne sur laquelle sont situées les encoches transverses $(18,28)$.

Selon Barnes (13), il existe des hypoplasies ou des aplasies de la pars basilaris causées par un défaut d'un ou des deux cartilages parachordaux, qui peuvent impacter ou non le foramen magnum. Les fissures sagittales proviennent d'un défaut de fusion incomplète des deux cartilages parachordaux, tandis que les fissures transverses unilatérale ou bilatérale seraient à l'origine d'un déplacement vers le haut de la limite occipito-vertébrale. Cela peut affecter les cartilages parachordaux en développement et produire ces encoches (13).

II n'y a donc actuellement pas de consensus sur l'origine exacte de ces encoches, même si tous les auteurs s'accordent sur le fait qu'elles apparaissent précocement.

Les encoches: syndrome génétique, syndrome pathologique ou variation osseuse asymptomatique?

La découverte d'encoches de la pars basilaris sur de très jeunes immatures du site médiéval italien "Quattro Macine » a été à l'origine d'une hypothèse sur 
leur caractère génétique. En effet, on a retrouvé des encoches bilatérales sur deux fœtus (estimés proches du terme) qui étaient inhumés dans une même sépulture. Leur apparentement a alors été évoqué, et l'origine génétique des encoches a été discutée par Anderson (19). Cependant, bien qu'elle soit plausible, cette hypothèse n'est pas privilégiée dans la littérature.

Plusieurs auteurs attribuent les encoches transverses à une variation osseuse asymptomatique présente au sein de plusieurs populations pour lesquelles on les retrouve à la fois chez des immatures et des adultes. Les encoches seraient alors formées par deux centres d'ossification incomplètement fusionnés au niveau de la crista synostotica $(18,28)$.

Les nombreux cas inventoriés dans l'étude ancienne de Le Double (14), tout comme le vocabulaire utilisé par l'auteur sont à prendre avec précautions. En effet, bien que les encoches ne soient pas spécifiquement attribuées à un syndrome pathologique ou à une variation osseuse asymptomatique, les termes employés renvoient quant à eux le plus souvent à une origine pathologique (14). Selon l'auteur, les segmentations seraient des défauts de soudure totale ou partielle du centre unique d'ossification avec d'autres centres anormaux présents dans le moule cartilagineux (14).

Ainsi, l'origine d'une variation osseuse asymptomatique peut être due à deux centres d'ossification de la pars basilaris incomplètement fusionnés $(17,47)$, au maintien de la limite entre le cartilage parachordal et les sclérotomes occipitaux $(27,28,44)$, ou au déplacement de la position normale de la limite occipito-vertébrale qui affecte le cartilage parachordal en développement (13) formant ainsi des encoches asymptomatiques. L'origine pathologique peut 
être due à un défaut de fusion partiel ou total du centre d'ossification unique de la pars basilaris avec d'autres centres anormaux (14), ou d'une atteinte des sclérotomes occipitaux $(15,48-51)$.

Enfin, plusieurs études font mention des encoches transverses chez des cas pathologiques. Un cas d'encoches bilatérales de la pars basilaris est décrit chez un fœtus prématuré présentant des malformations létales (15). Les encoches apparaissent également chez des individus porteurs d'anomalies chromosomiques. Une malformation de la pars basilaris a été observée chez dix fœtus âgés de 15 à 20 semaines d'aménorrhée porteurs de trisomie 18 (48). Pour six d'entre eux, une encoche unilatérale est observée, pour les trois autres une encoche bilatérale, et le dernier a une pars basilaris courte et triangulaire (48). Une encoche bilatérale a également été vue chez un fœtus de 20 semaines d'aménorrhée atteint de trisomie 21 (49), alors qu'il ne semble pas y avoir de malformation pour les trisomies 13 (52). Autres cas, des malformations de la pars basilaris ont été observées chez des fœtus âgés de 15 à 23 semaines atteints de triploïdie, avec pour la plupart de larges encoches unilatérales ou bilatérales au niveau de la partie antérieure (50). Un fœtus de 23 semaines a une pars basilaris séparée en deux éléments avec une encoche transverse complète (50). Enfin, on peut prendre l'exemple du syndrome de Cornelia de Lange pour lequel les individus atteints sont porteurs d'encoches complètes ou incomplètes (51).

Jusqu'à présent, il n'existait pas d'étude dans la littérature qui confrontait la 
morphologie des pars basilaris d'individus pathologiques et sains. D'après les résultats obtenus sur notre échantillon d'imagerie médicale, dont la condition pathologique ou non est clairement documentée, il n'est pas possible de conclure de manière formelle. Le nombre d'encoches (observées dans 2 cas sur 316 individus soit 0,63\% des individus sains et 3 cas sur 166 soit 1,81\% des individus pathologiques) est trop faible pour obtenir des résultats statistiquement interprétables. Nous retiendrons simplement de cette première approche que les encoches sont 2,9 fois plus fréquentes chez les individus pathologiques que chez les individus sains. On note également que les encoches d'origine pathologique peuvent être aussi bien unilatérales que bilatérales, et que le ratio entre la longueur des encoches et la largeur de la pars basilaris semble plus important pour les individus pathologiques que les individus sains, sans que nous puissions toutefois conclure à une valeur seuil.

\section{Conclusion}

Dans ce travail, nous avons prouvé sur deux échantillons d'effectifs importants (482 fœtus et nourrissons actuels étudiés par imagerie médicale, ainsi que 134 très jeunes immatures provenant de séries ostéo-archéologiques) que la fréquence des encoches transverses de la pars basilaris était faible (1,04\% pour l'échantillon d'imagerie médicale et $1,49 \%$ pour l'échantillon ostéo-archéologique) et qu'elles pouvaient aussi bien toucher des individus sains que pathologiques. De plus, il ne se dégage pas de typologie claire (atteinte unilatérale ou bilatérale) permettant de distinguer leur origine pathologique ou non, bien que le ratio longueur de l'encoche sur largeur de la pars basilaris semble plus élevé dans les 
cas pathologiques. Dans l'état actuel des connaissances, et en l'absence de conclusion plus formelle sur leur étiologie, il nous semble que leur découverte en contexte médico-légal ou bioarchéologique doit conduire les experts à nuancer leurs conclusions sur l'estimation de l'âge au décès, le risque d'une pathologie éventuelle étant de modifier la trajectoire ontogénique de l'enfant et par conséquent de biaiser l'estimation de l'âge basée sur des approches métriques.

\section{Remerciements}

Les auteurs souhaitent remercier Alain Badin de Montjoye (Conservateur territorial du Patrimoine honoraire, Conseil général de I'Isère, responsable d'opération de la fouille préventive Eglise Saint-Pierre, Moirans, Auvergne-Rhône-Alpes), Isabelle Daveau (ingénieur d'études INRAP, responsable d'opération de la fouille préventive 110 Chemin de la farigoule, Castelnau-Le-Lez, Occitanie) et Florence Parent (ingénieur d'études INRAP, responsable d'opération de la fouille préventive Chemin de Saint-Privat, Rousset, Provence-Alpes-Côte d'Azur) pour l'accès aux échantillons ostéo-archéologiques.

Conflits d'intérêts : aucun. 


\section{Références}

1. Fazekas IG, Kósa F. Forensic Fetal Osteology. Akadémiai Kiadó; 1978. 424 p.

2. Kyrkanides S, Kjaer I, Fischerhansen B. Development of the basilar part of the occipital bone in normal human fetuses. J Craniofac Genet Dev Biol. sept 1993;13(3):184-92.

3. Macklin CC. Preliminary note on the skull of a human fetus of $43 \mathrm{MM}$. Greatest length. Anat Rec. 1921;22(4):251-265.

4. Nemzek WR, Brodie HA, Hecht ST, Chong BW, Babcook CJ, Seibert JA. MR, CT, and Plain Film Imaging of the Developing Skull Base in Fetal Specimens. Am J Neuroradiol. 1 oct 2000;21(9):1699-706.

5. Noback CR, Robertson GG. Sequences of appearance of ossification centers in the human skeleton during the first five prenatal months. Am J Anat. juill 1951;89(1):1-28.

6. Noback CR. The developmental anatomy of the human osseous skeleton during the embryonic, fetal and circumnatal periods. Anat Rec. 1 janv 1944;88(1):91-125.

7. Scheuer L, Black S. The Juvenile Skeleton. London: Elsevier Academic Press; 2004. 496 p.

8. Jeffery N, Spoor F. Ossification and midline shape changes of the human fetal cranial base. Am J Phys Anthropol. 2004;123(1):78-90.

9. Scheuer L, Black S, Cunningham C. Developmental Juvenile Osteology. Academic Press; 2000. 599 p.

10. Kamina P, Martinet C. Anatomie clinique. 4e édition, Paris: Maloine; 2009.

11. Scheuer L, Black S, Cunningham C. Developmental Juvenile Osteology. San Diego: Elsevier Academic Press; 2000. 599 p.

12. Bories J. Le crâne humain : Ostéologie - Anatomie radiologique. Paris, France: Springer Science \& Business Media; 1996. 273 p.

13. Barnes E. Atlas of Developmental Field Anomalies of the Human Skeleton: A Paleopathology Perspective. 1 edition. Hoboken, N.J: Wiley-Blackwell; 2012. 232 p.

14. Le Double AF. Traité des variations des os du crane de l'homme, et de leur signification au point de vue de l'anthropologie zoologique. Préface d' Edmond Perrier [Internet]. Paris Vigot; 1903 [cité 24 août 2016]. 576 p. Disponible sur: http://archive.org/details/traitdesvariat00ledouoft

15. Johnson G, Israel H. Basioccipital Clefts. Radiology. 1979;133(1):101-3.

16. List CF. Neurologic syndromes accompanying developmental anomalies of occipital bone, atlas and axis. Arch Neurol Psychiatry. 1 avr 1941;45(4):577-616.

17. Hofmann E, Prescher A. The Clivus. Clin Neuroradiol. 1 juin 2012;22(2):123-39. 
18. Kawakubo Y, Dodo Y, Nara T, Kuraoka A. Transverse basilar cleft detected in prehistoric Jomon skulls from Japan. Anthropol Sci. avr 2014;122(1):45-50.

19. Anderson T. Basilar clefting: a familial condition? Ann Anat-Anat Anz. nov 2000;182(6):583-7.

20. Kruyff E. Transverse Cleft in the Basi-Occiput. Acta Radiol Diagn (Stockh). 1 janv 1967;6(1):41-8.

21. Rossi. Arch. p. l'ant. e la etnologia. 1891.

22. Albrecht $P$. Mémoire sur le basiotique, un nouvel os de la base du crâne. Communication présenté à: Société d'anthropologie de Bruxelles; 1883; Bruxelles.

23. Osawa G. On the basilar part of the occipital bone. [Kotokotsu kisobu ni tsuite.]. 1894;8:642-5.

24. Shima G. A study on the Mongolian skulls.[Mokojintokotsu no kenkyu.]. 1941;2:1-108.

25. Limson M. Observations on the bones of the skull in white and negro fetuses and infants. Contributions to Embryology. 1932;23:205-33.

26. Lombardi G. The occipital vertebra. Am J Roentgenol Radium Ther Nucl Med. août 1961;86:260-9.

27. Shapiro R, Robinson F. Embryogenesis of the human occipital bone. Am J Roentgenol. 1 mai 1976;126(5):1063-8.

28. Lang J. Skull Base and Related Structures: Atlas of Clinical Anatomy. Schattauer Verlag; 1995. $360 \mathrm{p}$.

29. Maresh MM. Linear growth of long bones of extremities from infancy through adolescence: continuing studies. AMA Am J Dis Child. 1955;89(6):725-742.

30. Maresh MM. Measurements from roentgenograms. In: Human growth and development. Springfield: McCammon RW, editor; 1970. p. 157-200. (II: CC Thomas).

31. Ubelaker DH. Human skeletal remains: excavation, analysis and interpretation. Chicago, Illinois: Aldine Publishing Co. Inc.; 1978. 116 p.

32. Adalian P. Evaluation multiparamétrique de la croissance foetale : application à la détermination de l'âge et du sexe. [Internet]. [Marseille, France]: Université de la Méditerranée - Aix-Marseille 2; 2001 [cité 5 avr 2018]. Disponible sur: http://www.theses.fr/2001AIX20692

33. Adalian P, Piercecchi-Marti MD, Bourliere-Najean B, Panuel M, Leonetti G, Dutour O. New foetal age determination formula. C R Biol. mars 2002;325(3):261-9.

34. Rigeade C. Chapelle Notre-Dame de la mer (Fos-sur-Mer, Bouches-du-Rhône), Bilan scientifique, D.R.A.C. - P.A.C.A. - S.R.A. 2014 p. 103-4. 
35. Daveau I. 110 Chemin de la Farigoule (Castelnau-Le-Lez, Hérault), Bilan scientifique, D.R.A.C. - Occitanie. 2016.

36. Parent $F$. Chemin de Saint-Privat, de la villa au(x) cimetière(s), en passant par la chaux (Provence-Alpes-Côte-d'Azur, Bouches-du-Rhône, Rousset), Rapport final d'opération, 3 volumes. Inrap Méditerranée; 2017 p. 953.

37. Diverrez F, Poulmarc'h M, Schmitt A. Nouvelles données sur les inhumations ad sanctos à l'époque moderne en milieu rural : le cas de l'église Saint-Pierre de Moirans (Isère). Bull Mém Société Anthropol Paris. 1 déc 2012;24(3):167-78.

38. Tzortzis S, Fiers $E$, Julien $M$, Pogneaux $N$, Adalian $P$, Ardagna $\mathrm{Y}$, et al. Un ensemble funéraire médiéval et moderne aux abords de la chapelle Saint-Jean (L'Argentière-laBessée, Hautes Alpes, France). Approches anthropologique et archéologique. Homme Ses Images Mes Représentations Constr Actes 25e Colloq GALF Marseille 16-18 Juillet 2001 Fac Médecine Marseille. 2004;364-78.

39. Coleman MN, Colbert MW. Technical note: CT thresholding protocols for taking measurements on three-dimensional models. Am J Phys Anthropol. mai 2007;133(1):723-5.

40. Spoor CF, Zonneveld FW, Macho GA. Linear measurements of cortical bone and dental enamel by computed tomography: Applications and problems. Am J Phys Anthropol. 1 août 1993;91(4):469-84.

41. R Core Team. R: A language and environment for statistical computing. R Foundation for Statistical Computing, Vienna, Austria. [Internet]. 2013. Disponible sur: http://www.R-project.org/

42. Smith HD. Observations on the occipital bones in a series of Egyptian skulls with especial reference to the persistence of the synchondrosis condylo-squamoza (Zaaijer; synchondrosis intraoccipital posterior, BNA). 1912;8:257-61.

43. Carlson BM. Human Embryology and Developmental Biology. 4th edition. Philadelphia, PA: Mosby; 2009. 560 p.

44. Hamilton WJ, Mossman HW. Human Embryology: Prenatal Development of Form and Function. 4th edition. Cambridge: W.Heffer \& Sons Ltd; 1972. 658 p.

45. Langman J, Sadler TW. Atlas d'embryologie médicale de Langman. Pradel. RueilMalmaison: Pradel, Wolters Kluwer France; 2008. 160 p.

46. Larsen W, Brauer PR, Schoenwolf GC, Francis-West P. Embryologie humaine. De Boeck Superieur; 2017. 563 p.

47. Bryce TH. Osteology and arthrology. In: Quain's Elements of Anatomy. 11th edn. London: Longmans, Green; 1915. p. 1-329.

48. Kjaer I, Keeling JW, Hansen BF. Pattern of malformations in the axial skeleton in human trisomy 18 fetuses. Am J Med Genet. 11 nov 1996;65(4):332-6. 
49. Keeling JW, Hansen BF, Kjaer I. Pattern of malformations in the axial skeleton in human trisomy 21 fetuses. Am J Med Genet. 11 févr 1997;68(4):466-71.

50. Sonnesen L, Nolting D, Engel U, Kjaer I. Cervical vertebrae, cranial base, and mandibular retrognathia in human triploid fetuses. Am J Med Genet A. févr 2009;149A(2):177-87.

51. Whitehead MT, Nagaraj UD, Pearl PL. Neuroimaging features of Cornelia de Lange syndrome. Pediatr Radiol. 1 août 2015;45(8):1198-205.

52. Kjaer I, Keeling JW, Hansen BF. Pattern of malformations in the axial skeleton in human trisomy 13 fetuses. Am J Med Genet. 27 juin 1997;70(4):421-6. 


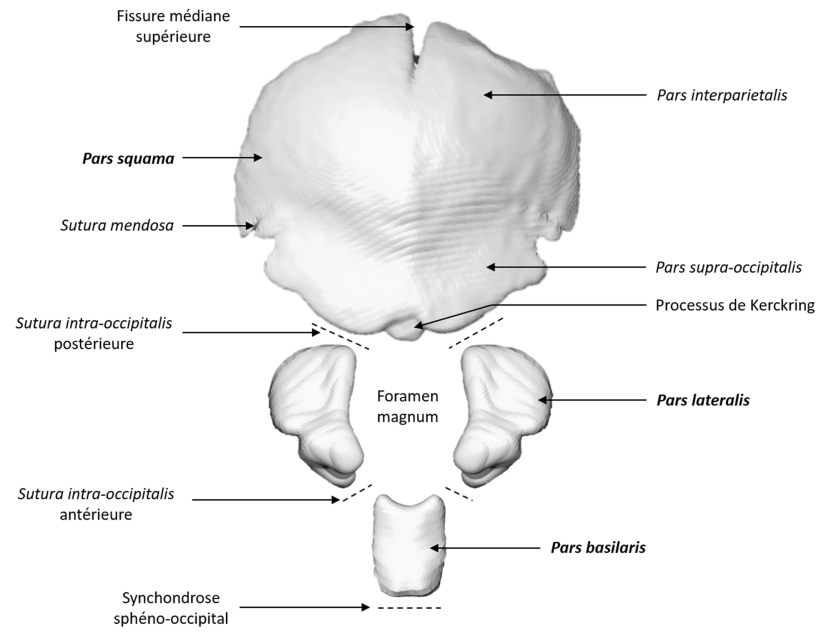




\section{Bord antérieur du foramen magnum}

Articulation avec la pars lateralis

Articulation avec la pars petrosa de l'os temporal

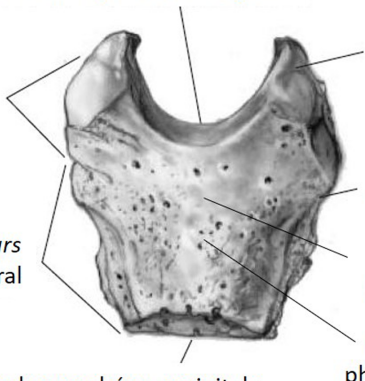

Synchondrose sphéno-occipitale
$1 / 3$ du condyle occipital

Angle latéral

Tubercule pharyngien

Fossette pharyngienne 

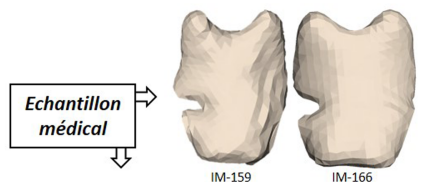

\section{Encoches unilatérales non pathologiques}

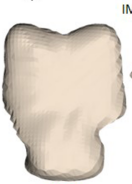

IM-159

IM-166

IM-327

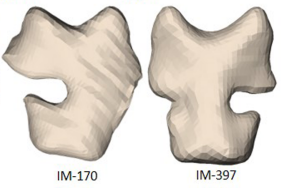

Encoches unilatérales pathologiques

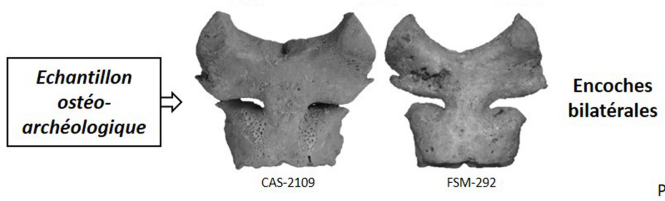

